\title{
88. A Case of Triplets in Shirakawa
}

\author{
By Yoshito Furuya, Takashi Tanaka, and Mitsuo Yokoyama \\ Department of Legal Medicine, School of Medicine, Tokyo Medical \\ and Dental University \\ (Comm. by T. FuruhatA, M.J.A., June 12, 1958)
}

The present report is concerned with the observation on blood groups and types, fingerprint patterns, palm patterns, and soleprint patterns of triplets, born in Shirakawa, Fukushima prefecture.

They were born on Feb. 19, 1955. The mother (S. K.), age 32, and her husband (K. K.), age 37, are both in normal health. There are no hereditary multiple pregnancy and malformation in the family before.

They have a daughter, 9 years old. The triplets were born in the second delivery. In spite of being a premature delivery (the term of pregnancy: 32 weeks), the triplets are all well now (Table I).

Table I. Observations on the children

\begin{tabular}{|c|c|c|c|c|c|c|c|c|c|}
\hline & \multicolumn{2}{|c|}{$\begin{array}{l}\text { Gener- } \\
\text { ation }\end{array}$} & & Name & Age & Sex & Height & Weight & $\begin{array}{l}\text { Circum- } \\
\text { ference } \\
\text { of chest }\end{array}$ \\
\hline 1st child & & 2 & Yoshio & KAWAGUCHI & 3 & $\hat{\sigma}$ & $97.8 \mathrm{~cm}$ & $17 \mathrm{~kg}$ & $55.5 \mathrm{~cm}$ \\
\hline 2nd child & $\mathrm{F}_{\text {II }}$ & 3 & Kin-ya & KAWAGUCHI & 3 & $\hat{0}$ & $97 \mathrm{~cm}$ & $15.5 \mathrm{~kg}$ & $55 \mathrm{~cm}$ \\
\hline 3rd child & & 4 & Yasuhi & iro KAWAGUCHI & 3 & $\hat{0}$ & $94 \mathrm{~cm}$ & $14.8 \mathrm{~kg}$ & $50 \mathrm{~cm}$ \\
\hline
\end{tabular}

\begin{tabular}{|c|c|c|c|c|c|c|}
\hline $\begin{array}{c}\text { Sitting } \\
\text { height }\end{array}$ & Character & $\begin{array}{c}\text { Ear- } \\
\text { wax }\end{array}$ & $\begin{array}{c}\text { Mid-digital } \\
\text { hair }\end{array}$ & $\begin{array}{c}\text { Taste-ability } \\
\text { for phenyl- } \\
\text { thio-carbamide }\end{array}$ & $\begin{array}{c}\text { Handed- } \\
\text { ness }\end{array}$ & Illness \\
\hline $57 \mathrm{~cm}$ & vigorous & wet & $(-)$ & $(-)$ & right & $\begin{array}{c}\text { Hernia inguinalis } \\
\text { bilateralis }\end{array}$ \\
\hline $57 \mathrm{~cm}$ & vigorous & wet & $(-)$ & $(-)$ & right & $\begin{array}{c}\text { Hernia inguinalis } \\
\text { bilateralis }\end{array}$ \\
\hline $55.5 \mathrm{~cm}$ & meek & wet & $(-)$ & $(-)$ & right & $(-)$ \\
\hline
\end{tabular}

All the family members were tested for the following blood group systems, i.e. ABO, MN, Q, ST, Lewis, Rh-Hr, Kell, and Duffy. They are all of the " $\mathrm{O}$ " blood group. The blood groups and types of two of the triplets (II-2, II-3) are identical, but the third triplet (II-4) has different types of MN and Rh-Hr blood types than the other (II-2, II-3). 
Table II. Blood groups and types of the family

\begin{tabular}{|c|c|c|c|c|c|c|c|}
\hline \multicolumn{2}{|c|}{$\begin{array}{l}\text { Genera- } \\
\text { tion }\end{array}$} & Name & Age & $\operatorname{Sex}$ & $\begin{array}{l}\text { ABO blood } \\
\text { group }\end{array}$ & $\begin{array}{l}\text { MN blood } \\
\text { type }\end{array}$ & $\begin{array}{l}Q \text { blood } \\
\text { type }\end{array}$ \\
\hline \multirow{2}{*}{$\mathrm{F}_{\mathrm{I}}$} & 1 & Kenji KawAGUCHI & 37 & $\hat{\sigma}$ & $\mathrm{O}$ & $\mathrm{MN}$ & q \\
\hline & 2 & Shizuko KaWAGUCHI & 32 & 우 & $\mathrm{O}$ & $\mathrm{MN}$ & q \\
\hline \multirow{4}{*}{$\mathrm{F}_{\text {II }}$} & 1 & Yûko KaWAGUCHI & 9 & 우 & $\mathrm{O}$ & $\mathrm{N}$ & $q$ \\
\hline & 2 & Yoshio KawaguCHI & 3 & $\hat{\delta}$ & $\mathrm{O}$ & M & $q$ \\
\hline & 3 & Kin-ya KAWAGUCHI & 3 & $\hat{o}$ & $\mathrm{O}$ & M & $q$ \\
\hline & 4 & Yasuhiro KawaGUCHI & 3 & $\uparrow$ & $\mathrm{O}$ & $\mathrm{N}$ & $q$ \\
\hline
\end{tabular}

\begin{tabular}{c|c|c|c|c|c|}
\hline $\begin{array}{c}\text { S and T blood } \\
\text { system }\end{array}$ & $\begin{array}{c}\text { Lewis blood } \\
\text { system }\end{array}$ & $\begin{array}{c}\text { Rh-Hr } \\
\text { blood type }\end{array}$ & $\begin{array}{c}\text { Kell blood } \\
\text { type }\end{array}$ & $\begin{array}{c}\text { Duffy blood } \\
\text { type }\end{array}$ & Agglutinin \\
\hline St & Le (a-) & CC:D:ee & kk & Fy (a+) & $\alpha: 64 \times \beta: 64 \times$ \\
\hline St & Le (a-) & Cc:D:Ee & $\mathrm{kk}$ & Fy (a+) & $\alpha: 256 \times \beta: 256 \times$ \\
\hline St & Le (a-) & Cc:D:Ee & $\mathrm{kk}$ & Fy (a+) & $\alpha: 256 \times \beta: 256 \times$ \\
\hline St & Le (a-) & CC:D:ee & $\mathrm{kk}$ & Fy (a+) & $\alpha: 64 \times \beta: 128 \times$ \\
\hline St & Le (a-) & CC:D:ee & $\mathrm{kk}$ & Fy (a+) & $\alpha: 64 \times \beta: 128 \times$ \\
\hline St & Le (a-) & Cc:D:Ee & $\mathrm{kk}$ & Fy (a+) & $\alpha: 64 \times \beta: 64 \times$ \\
\hline
\end{tabular}

The fingerprint patterns, ${ }^{*}$ palm patterns, ${ }^{* *}$ and soleprint patterns***) are shown in Tables III, IV, and V. In these investigations, it was observed that two of the triplets (II-2, II-3) are exactly alike in all respects, but the other triplet (II-4) is slightly different in some respects.

According to these observations, the first child (II-2) and the second child (II-3) are proved to be monozygotic twins, while the third child (II-4) is proved to be dizygotic from each of the other two. Therefore, the triplets are proved to be dizygotic.

*) Fingerprints are classified into three groups, an arch (A), a loop (L), and a whorl (W). The loop is divided into two types, a radial loop (R) and an ulnar loop (U). The whorl is divided into three types, inner, meeting, and outer. The biological value of fingerprints (b.V.) and its genotypes are calculated according to Matsukura's1)-6) genetic classification (1950). Furthermore, the genotypes are determined by calculation of papillary ridges using Matsukura's ${ }^{7 / 8)}$ method (1952).

**) Palm patterns are classified by using Cummins-Midlo's ${ }^{9)}$ method.

$* * *)$ Soleprint patterns are classified by using Wentworth-Wilder's ${ }^{10)}$ method. 


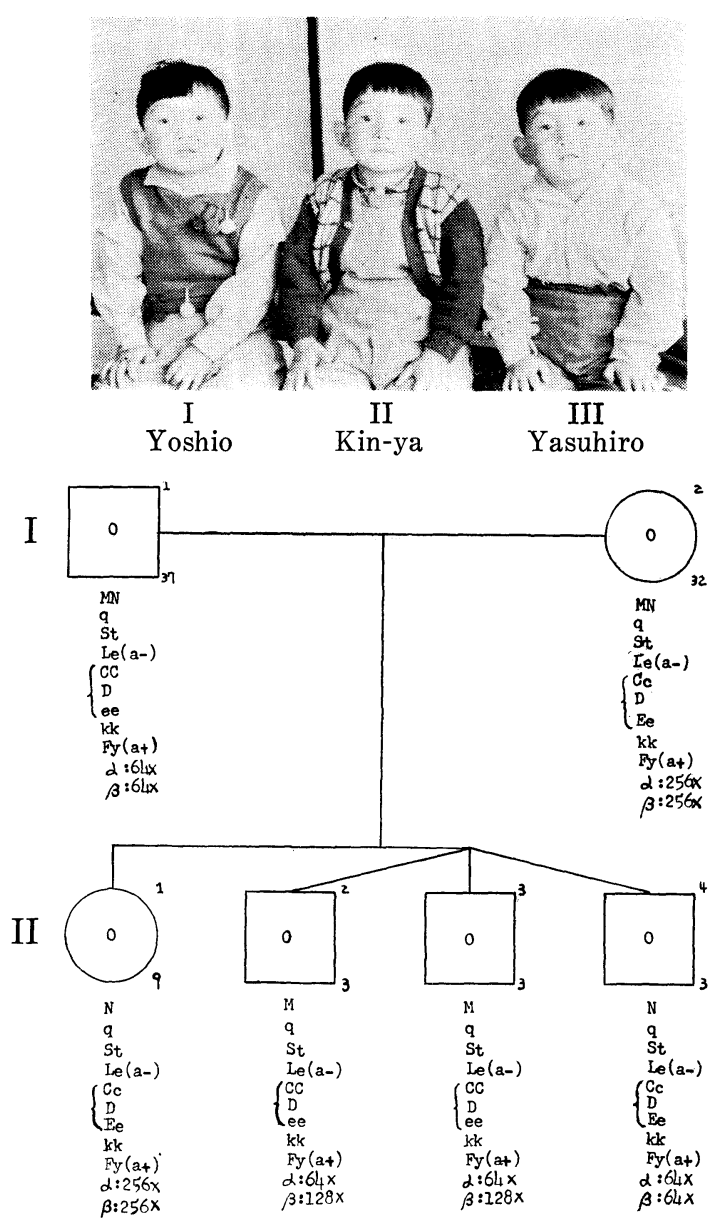

Fig. 1. A family line of KAWAGUCHI (blood groups and types)

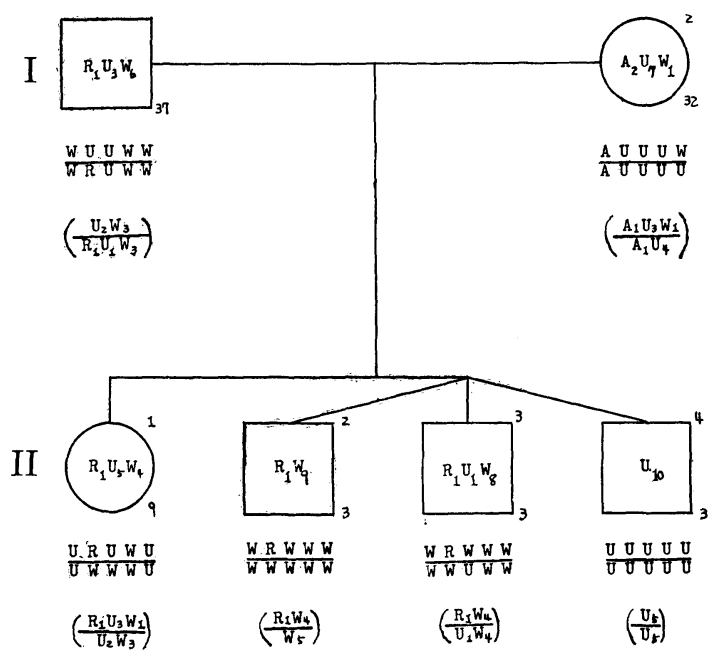

Fig. 2. A family line of KAWAGUCHI (fingerprints) 


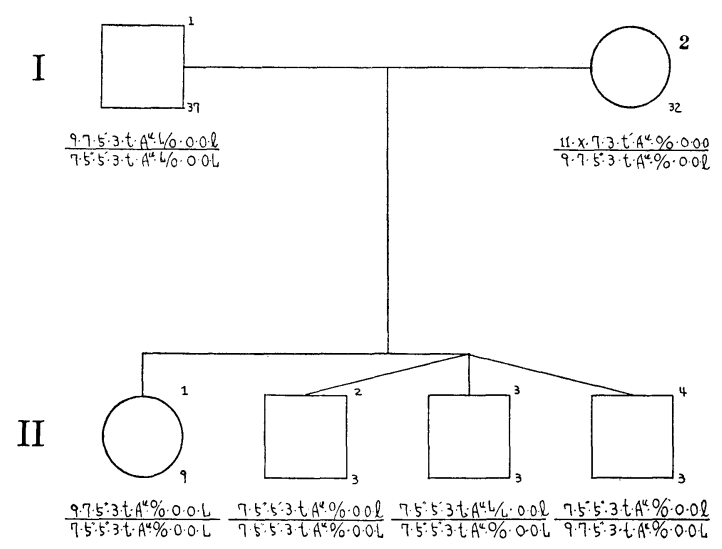

Fig. 3. A family line of KAWAGUCHI (handprints)

Table III. Fingerprint patterns

\begin{tabular}{|c|c|c|c|c|c|c|c|c|c|c|}
\hline \multicolumn{2}{|c|}{$\begin{array}{c}\text { Genera- } \\
\text { tion }\end{array}$} & Name & Age & Sex & & $\underset{\text { (1st) }}{\text { Thumb }}$ & $\begin{array}{l}\text { Index } \\
\text { finger } \\
\text { (2nd) }\end{array}$ & $\begin{array}{c}\text { Middle } \\
\text { finger } \\
\text { (3rd) }\end{array}$ & $\begin{array}{l}\text { Ring } \\
\text { finger } \\
\text { (4th) }\end{array}$ & $\begin{array}{r}\text { Little } \\
\text { finger } \\
\text { (5th) }\end{array}$ \\
\hline \multirow{2}{*}{$\mathrm{F}_{\mathrm{I}}$} & 1 & Kenji KaWAGUCHI & 37 & $\hat{0}$ & $\begin{array}{l}\mathrm{L} \\
\mathrm{R}\end{array}$ & $\begin{array}{l}\text { W } \\
W\end{array}$ & $\begin{array}{l}\mathrm{U} \\
\mathrm{R}\end{array}$ & $\begin{array}{l}\mathrm{U} \\
\mathrm{U}\end{array}$ & $\begin{array}{l}\text { W } \\
W\end{array}$ & $\begin{array}{l}\mathrm{W} \\
\mathrm{W}\end{array}$ \\
\hline & 2 & Shizuko KAWAGUCHI & 32 & 우 & $\begin{array}{l}\mathrm{L} \\
\mathrm{R}\end{array}$ & $\begin{array}{l}\mathrm{A} \\
\mathrm{A}\end{array}$ & $\begin{array}{l}\mathrm{U} \\
\mathrm{U}\end{array}$ & $\begin{array}{l}\mathrm{U} \\
\mathrm{U}\end{array}$ & $\begin{array}{l}\mathrm{U} \\
\mathrm{U}\end{array}$ & $\begin{array}{l}\mathrm{W} \\
\mathrm{U}\end{array}$ \\
\hline \multirow{4}{*}{$\mathrm{F}_{\text {II }}$} & 1 & Yûko KAwAGUCHI & 9 & 우 & $\begin{array}{l}\mathrm{L} \\
\mathrm{R}\end{array}$ & $\begin{array}{l}\mathrm{U} \\
\mathrm{U}\end{array}$ & $\begin{array}{l}\mathrm{R} \\
\mathrm{W}\end{array}$ & $\begin{array}{l}\mathrm{U} \\
\mathrm{W}\end{array}$ & $\begin{array}{l}\mathrm{W} \\
\mathrm{W}\end{array}$ & $\begin{array}{l}\mathrm{U} \\
\mathrm{U}\end{array}$ \\
\hline & 2 & Yoshio KaWAGUCHI & 3 & 今 & $\begin{array}{l}\mathrm{L} \\
\mathrm{R}\end{array}$ & $\begin{array}{l}\mathrm{W} \\
\mathrm{W}\end{array}$ & $\begin{array}{l}\mathrm{R} \\
\mathrm{W}\end{array}$ & $\begin{array}{l}\mathrm{W} \\
\mathrm{W}\end{array}$ & $\begin{array}{l}\mathrm{W} \\
\mathrm{W}\end{array}$ & $\begin{array}{l}\mathrm{W} \\
\mathrm{W}\end{array}$ \\
\hline & 3 & Kin-ya KAWAGUCHI & 3 & $\hat{\sigma}$ & $\begin{array}{l}\mathrm{L} \\
\mathrm{R}\end{array}$ & $\begin{array}{l}\mathrm{W} \\
\mathrm{W}\end{array}$ & $\begin{array}{l}\mathrm{R} \\
\mathrm{W}\end{array}$ & $\begin{array}{l}\text { W } \\
\text { U }\end{array}$ & $\begin{array}{l}\mathrm{W} \\
\mathrm{W}\end{array}$ & $\begin{array}{l}\mathrm{W} \\
\mathrm{W}\end{array}$ \\
\hline & 4 & Yasuhiro KAwAGUCHI & 3 & $\hat{0}$ & $\begin{array}{l}\mathrm{L} \\
\mathrm{R}\end{array}$ & $\begin{array}{l}\mathrm{U} \\
\mathrm{U}\end{array}$ & $\begin{array}{l}\mathrm{U} \\
\mathrm{U}\end{array}$ & $\begin{array}{l}\mathrm{U} \\
\mathrm{U}\end{array}$ & $\begin{array}{l}\mathrm{U} \\
\mathrm{U}\end{array}$ & $\begin{array}{l}\mathrm{U} \\
\mathrm{U}\end{array}$ \\
\hline
\end{tabular}

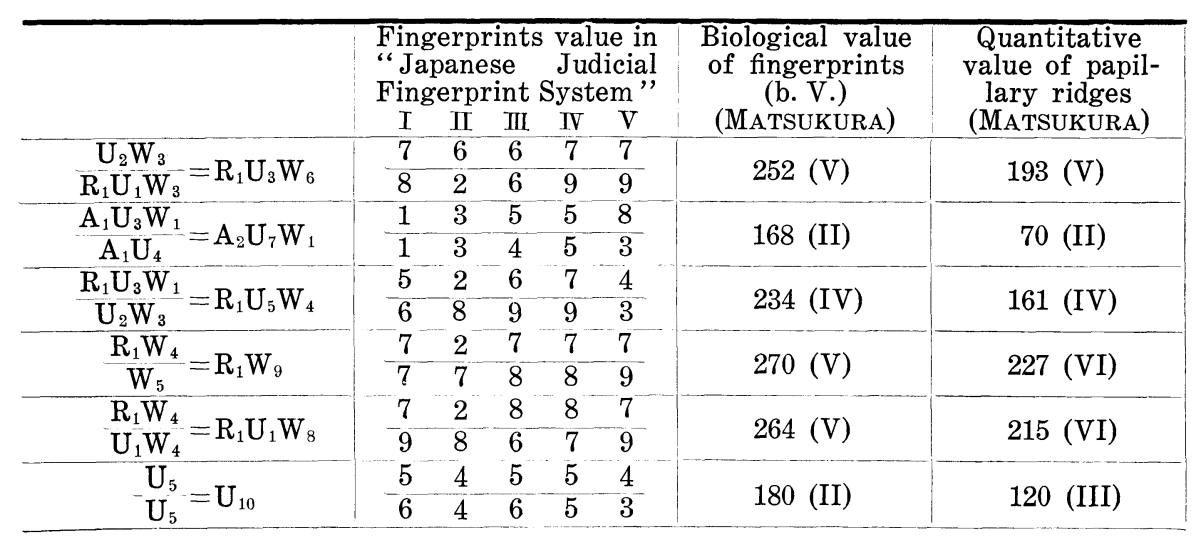


Table IV. Palm patterns

\begin{tabular}{|c|c|c|c|c|}
\hline \multicolumn{2}{|c|}{$\begin{array}{l}\text { Genera- } \\
\text { tion }\end{array}$} & Name & Age & Sex \\
\hline \multirow{2}{*}{$\mathrm{F}_{\mathrm{I}}$} & 1 & Kenji KaWAGUCHI & 37 & $\hat{o}$ \\
\hline & 2 & Shizuko KaWAGUCHI & 32 & 오 \\
\hline \multirow{4}{*}{$F_{I I}$} & 1 & Yûko KAWAGUCHI & 9 & 우 \\
\hline & 2 & Yoshio KaWAGUCHI & 3 & $\hat{\delta}$ \\
\hline & 3 & Kin-ya KAWAGUCHI & 3 & 今. \\
\hline & 4 & Yasuhiro KAWAGUCHI & 3 & $\hat{0}$ \\
\hline
\end{tabular}

\begin{tabular}{|c|c|}
\hline \multicolumn{2}{|c|}{ Handprints } \\
\hline Left hand & Right hand \\
\hline $9 \cdot 7 \cdot 5^{\prime} \cdot 3 \cdot t \cdot \mathrm{A}^{\mathrm{u}} \cdot \mathrm{L} / 0 \cdot 0 \cdot 0 \cdot l$ & $7 \cdot 5^{\prime \prime} \cdot 5^{\prime} \cdot 3 \cdot t \cdot \mathrm{A}^{\mathrm{u}} \cdot \mathrm{L} / 0 \cdot 0 \cdot 0 \cdot \mathrm{L}$ \\
\hline $11 \cdot x \cdot 7 \cdot 3 \cdot t^{\prime} \cdot \mathrm{A}^{\mathrm{u}} \cdot \mathrm{O} / 0 \cdot 0 \cdot 0 \cdot 0$ & $9 \cdot 7 \cdot 5^{\prime \prime} \cdot 3 \cdot t \cdot \mathrm{A}^{\mathrm{u}} \cdot \mathrm{O} / 0 \cdot 0 \cdot 0 \cdot l$ \\
\hline $9 \cdot 7 \cdot 5^{\prime \prime} \cdot 3 \cdot t \cdot \mathrm{A}^{\mathrm{u}} \cdot \mathrm{O} / 0 \cdot 0 \cdot \mathrm{O} \cdot \mathrm{L}$ & $7 \cdot 5^{\prime \prime} \cdot 5^{\prime \prime} \cdot 3 \cdot t \cdot \mathrm{A}^{\mathrm{u}} \cdot \mathrm{O} / \mathrm{O} \cdot \mathrm{O} \cdot \mathrm{O} \cdot \mathrm{L}$ \\
\hline $7 \cdot 5^{\prime \prime} \cdot 5^{\prime} \cdot 3 \cdot t \cdot A^{u} \cdot 0 / 0 \cdot 0 \cdot 0 \cdot l$ & $7 \cdot 5^{\prime \prime} \cdot 5^{\prime} \cdot 3 \cdot t \cdot \mathrm{A}^{\mathrm{u}} \cdot \mathrm{O} / \mathrm{O} \cdot 0 \cdot \mathrm{O} \cdot \mathrm{L}$ \\
\hline $7 \cdot 5^{\prime \prime} \cdot 5^{\prime} \cdot 3 \cdot t \cdot \mathrm{A}^{\mathrm{u}} \cdot \mathrm{L} / \mathrm{L} \cdot 0 \cdot O \cdot l$ & $7 \cdot 5^{\prime \prime} \cdot 5^{\prime \prime} \cdot 3 \cdot t \cdot \mathrm{A}^{\mathrm{u}} \cdot \mathrm{O} / \mathrm{O} \cdot 0 \cdot 0 \cdot \mathrm{L}$ \\
\hline $7 \cdot 5^{\prime \prime} \cdot 5^{\prime \prime} \cdot 3 \cdot t \cdot \mathrm{A}^{\mathrm{u}} \cdot 0 / 0 \cdot 0 \cdot 0 \cdot l$ & $9 \cdot 7 \cdot 5^{\prime \prime} \cdot 3 \cdot t \cdot \mathrm{A}^{\mathrm{u}} \cdot \mathrm{O} / 0 \cdot 0 \cdot 0 \cdot \mathrm{L}$ \\
\hline
\end{tabular}

Table V. Soleprint patterns

\begin{tabular}{|c|c|c|c|c|c|}
\hline & \multirow{2}{*}{ Name } & \multirow{2}{*}{ Age } & \multirow{2}{*}{ Sex } & \multicolumn{2}{|c|}{ Soleprints } \\
\hline & & & & Left foot & Right foot \\
\hline 1st child & Yoshio KaWAGUCHI & 3 & 金 & $\mathrm{W} \cdot 0 \cdot 0 \cdot 0$ & $\mathrm{~W} \cdot 0 \cdot 0 \cdot 0$ \\
\hline 2nd child & Kin-ya KawaGUCHI & 3 & $\hat{0}$ & $\mathrm{~W} \cdot 0 \cdot 0 \cdot 0$ & $\mathrm{~W} \cdot 0 \cdot 0 \cdot 0$ \\
\hline 3rd child & Yasuhiro KAwAGUCHI & 3 & $\hat{0}$ & $W \cdot O \cdot U \cdot O \cdot d$ & $W \cdot \Omega \cdot U \cdot O \cdot d$ \\
\hline
\end{tabular}

\section{References}

1) Matsukura, T.,: On the biological classification of fingerprints and the "biological value of fingerprints (b.V.)", Jap. J. Leg. Med., 4 (3-4), 140-141 (1950).

2) Matsukura, T.,: On the biological value of fingerprints of parents and children, Jap. J. Leg. Med., 4 (3-4), 141-142 (1950).

3) Matsukura, T.,: On the genetic analysis of my "biological value of fingerprints (b.V.)", Jap. J. Leg. Med., 5 (6), 345 (1951).

4) Matsukura, T.,: On the subtypes of my biological classification of fingerprints, Jap. J. Leg. Med., 5 (6), 345-346 (1951).

5) Matsukura, T.,: Studies on the inheritance of fingerprints. I. A biological classification of fingerprints and an introduction of "the biological value of fingerprints", Shikoku acta med, 3 (1), 1-22 (1952).

6) Matsukura, T.,: Studies on the inheritance of fingerprints. II. Genetic analysis of "the biological value of fingerprints", Shikoku acta med, 3 (2), 55-68 (1952).

7) Matsukura, T.,: Studies on the inheritance of fingerprints. III. On the inheritance of the quantity of papillary ridges, Shikoku acta med, 3 (3), 108-119 (1952).

8) Matsukura, T.,: On the quantitative papillary line value of fingerprints, Jap. J. Leg. Med., 6 (3-4), 139 (1952).

9) Cummins, H., Midlo, C., et al.: Revised methods of interpreting and formulating palmar dermatoglyphics, Am. J. Phys. Anthrop., 12 (3), 415-473 (1929).

10) Wentworth, B., and Wilder, H. H.,: Personal Identification, 155-180 (1932). 